\title{
The feasibility and diagnostic accuracy by multiple cardiac biomarkers in emergency chest pain patients: a clinical analysis to compare 290 suspected acute coronary syndrome cases stratified by age and gender in Taiwan
}

Chung-Lieh Hung ${ }^{1,2}$, Ding-Kuo Chien ${ }^{3,4,5,6}$, Shou-Chuan Shih ${ }^{2,4,7}$ and Wen-Han Chang ${ }^{2,3,4,5,6,8^{*}}$

\begin{abstract}
Background: Accurate diagnosis of acute coronary syndrome (ACS) in a timely fashion is challenging in the elderly population, especially elderly women, who usually exhibit atypical clinical symptoms. A multiple cardiac biomarker (MCB) based approach has been shown to improve diagnostic efficacy of ACS. However, data in various age groups and sex differences remain largely unexplored.

Methods: Point-of-care testing (POCT) was performed on 290 patients (aged $\geq 18$ years) who were admitted to the emergency department (ED) with symptoms of acute chest pain under suspicion of acute coronary syndrome (ACS). The MCB approach in current work assessed four cardiac biomarkers: myoglobin, troponin I, creatine kinase-myocardial band isoenzyme fraction (CK-MB), and brain natriuretic peptide (BNP).

Results: Overall, the MCB approach demonstrated considerably higher sensitivity for elderly patients than for younger patients in identifying ACS (80.0\% [64.1-90.0] vs. $52.6 \%$ [37.3-67.5] for $\geq 65$ years and <65 years groups), with younger population showed greater specificity (44.1\% [35.3-53.4] vs. $84.9 \%$ [76.9-90.5] for $\geq 65$ years and <65 years groups, respectively). The highest sensitivity achieved for elderly women who reported chest pain was $87.5 \%$ [95 \% Cl: 64-96.5]). In general, the sensitivity of this approach was higher for female patients than for male patients (80\% [58.4-91.9] vs. $61 \%$ [47.8-73.0]).
\end{abstract}

Conclusions: The MCB approach can provide a quick and accurate clinical diagnosis in elderly and female patients, both of whom have traditionally proven to be challenging to diagnose from suspected acute coronary syndrome.

Keywords: Multiple cardiac biomarker, Acute coronary syndrome, Elderly

\footnotetext{
* Correspondence: branden888@gmail.com

${ }^{2}$ Department of Medicine, Mackay Medical College, New Taipei city, Taiwan

${ }^{3}$ Emergency Department, MacKay Memorial Hospital, Taipei, Taiwan

Full list of author information is available at the end of the article
} 


\section{Background}

Acute coronary syndrome (ACS) is an extremely common condition that is one of the leading causes of death worldwide. ACS is commonly encountered in the emergency room and occurs most often in elderly patients [1, 2]; however, in recent years the average age of patients with ACS has declined due to changes in lifestyle and personal behavior.

Women who have ACS differ from their male counterparts both in terms of reported symptoms, clinical manifestations and diagnosis rates [3-5]. In terms of symptoms and clinical manifestations, female patients primarily exhibit unstable angina and non-ST-segment elevation myocardial infarction (NSTEMI), while male patients primarily exhibit STEMI $[3,5]$. Regarding diagnosis, biomarker sensitivity and specificity in exercise tests have been reported to be lower in both women and men [5].

Cardiac biomarkers for acute coronary syndrome play key roles diagnosis, risk assessment, treatment, and supervision. Troponin I is a sensitive indicator of cardiomyocyte necrosis and an important biomarker for monitoring treatment. Myoglobin is the earliest biomarker of myocardial injury to appear, allowing doctors to rule out the possibility of myocardial infarction in a timely fashion. Apart from diagnostic role in heart failure, brain natriuretic peptide (BNP) is also closely related to acute coronary syndrome [6]. However, when used individually, these biomarkers are limited by several factors in clinical scenarios, especially in elderly patients [2].

Owing to differences in the diagnostic ability and performance of cardiac biomarkers across a wide range of patient population with age and gender distributions, and considering their important role in the diagnosis of acute coronary syndrome, we currently examined the accuracy of four cardiac biomarkers in the diagnosis of acute coronary syndrome in patients who experienced chest pain.

\section{Methods}

\section{Patient enrollment}

From March to November 2009, patients who visited Linkou Chang Gung Memorial Hospital, Taipei Mackey Memorial Hospital, Far Eastern Memorial Hospital, and China Medical University Hospital in Taiwan, with a primary complaint of chest pain and who were suspected of having acute coronary syndrome were recruited into this study. All enrolled patients were aged 18 years or older. Inclusion criteria includes: 1 ) adults $\geq 18$ years of age and consented to participate in the study group, 2) admitted to the emergency room due to chest pains or potential acute coronary syndrome symptoms, 3) symptoms unclear or atypical but suspected to be ACS requiring further electrocardiogram analysis and blood tests. Exclusion criteria includes: 1) Pregnancy, or clinical scenarios that may cause false positive test results of elevated Troponin I or BNP, such as skeletal myopathies, or renal insufficiency $[7,8]$ (defined by creatinine $>2.5 \mathrm{mg} / \mathrm{dL}$ ), 2) Non-Taiwan residents, patients whose contact information is unavailable, patients planning to go abroad and thus would be unavailable for follow-up, and patients who had already spent more than two hours in the emergency room reception area, 3) Patients who were transferred from other hospitals, 4) Cases where written permission could not be obtained or refused to participate the study, 5) Cases in whom the symptoms were already confirmed as not the cause of acute coronary syndrome, including pneumothorax, acute pulmonary embolism, aortic dissection, acute gastroenterological diseases or chronic obstructive lung disorder, 6) Initial electrocardiogram (ECG) had shown elevated ST segment or new LBBB. Subjects with ST elevation and new left bundle branch block (LBBB) were excluded since these clearly showed the onset of AMI, and therefore did not require diagnosis by MCB test systems. Researchers then examined patients' medical records and conducted telephone interviews to identify any subsequent health issues or discomfort experienced within 45 days after diagnosis. Within 45 days of treatment, cardiologists diagnosed patients with one of the following conditions as positive for acute coronary syndrome: ST-segment elevation myocardial infarction (STEMI); non-ST segment elevation myocardial infarction (NSTEMI); sudden cardiac arrest, high-grade atrioventricular block (AVB) or ventricular arrhythmia with cardiac catheter-proven coronary occlusion by thrombus formation; emergency, urgent, or elective revascularization; and unstable angina. All study participants underwent cardiac catheter during admission to confirm the diagnosis of acute coronary syndrome by experienced interventionalists/cardiologists and patients without these conditions were not enrolled in the study.

\section{Biochemical analysis and multi cardiac biomarker panel test}

During the examination, myoglobin, creatine kinasemyocardial band isoenzyme fraction (CK-MB), troponin I, and BNP were tested using a MCB CardioProfiler Panel (Alere, San Diego, CA). Blood (3 ml) was sampled from each patient using EDTA K2 Vacuette tubes. Next, $0.25 \mathrm{ml}$ of blood was siphoned from the total sample using a pipette and placed onto a test panel. The MCB test panel was then placed into a Triage MeterPro (Alere, San Diego, CA) for examination, the results of which were automatically displayed and printed after $15 \mathrm{~min}$. Data were presented in quantitative form with continuous variables presented. All results were blinded to investigators. Biomarker reference values (CardioProfiler Panel) were as follows: Troponin $I>$ $0.4 \mathrm{ng} / \mathrm{mL}, \mathrm{CK}-\mathrm{MB}>4.3 \mathrm{ng} / \mathrm{mL}$, myoglobin $>107 \mathrm{ng} / \mathrm{mL}$, and $\mathrm{BNP}>100 \mathrm{pg} / \mathrm{mL}$. 


\section{Statistical analysis}

Quantitative data were expressed as mean \pm standard deviation. Parametric data were compared by $t$ test, and categorical data were analyzed by the Chi-square test with Yates' correction or Fisher's exact test. Data were analyzed using SPSS $^{\circ}$ statistics software (SPSS 11.5; SPSS Inc., Chicago, IL, USA). Data were analyzed for sensitivity, specificity, positive and negative predictive values (PPV and NPV), and positive and negative likelihood ratios (LR+ and LR-). All $P<0.05$ was considered statistically significant with two-sided analysis with $P<0.05$ was considered statistically significant.

\section{Results}

A total of 290 patients with chest pain were enrolled in the current study with an average age of 64.5 years. Of the 290 patients, 146 were elderly ( $\geq 65$ years), with an average age of 75.4 years, which represented $50.3 \%$ of the total sample. The remaining 144 patients were $<65$ years of age (average age: 53.4 years) which represented $49.7 \%$ of the total sample. Further, 109 patients were women (71 elderly and 38 young), and constituted $37.6 \%$ of the total sample (Table 1).

A prior history of cardiovascular diseases (including prior stroke, myoacrdial infarction, history of coronary interventions or bypass graft surgery) was reported in
$34.5 \%$ of the participants $(100 / 290)$. Other common comorbidities included angina (30 \% [87/290]) and coronary angioplasty (22.5\% [54/290]). At baseline, elderly patients reported statistically significantly more medical conditions such as myocardial infarction, cardiovascular disease, heart failure, and stroke, compared with young patients $(p<0.05)$.

High blood pressure was found to be the most prevalent risk factor in patients with chest pain $(60.7 \%)$, followed by diabetes (30.7 \%), and dyslipidemia (30.7 \%). A larger proportion of elderly patients had high blood pressure and diabetes, while younger patients were more likely to have dyslipidemia, a family history of cardiovascular disease, or a history of smoking (Table 1).

A total of $73(25.2 \%)$ patients were diagnosed with cardiovascular disease, 35 of which were elderly (23.9\%) and 38 were young (26.4\%). Using the $\mathrm{MCB}$ approach in the diagnosis of acute coronary syndrome, young patients demonstrated a higher relative risk for acute coronary syndrome than elderly patients (6.25 vs. 3.16) (Table 2). In addition, 20 out of 109 (18.3\%) women were diagnosed with 53 out of 181 men $(29.3 \%)$. The MCB approach in the diagnosis of acute coronary syndrome revealed a higher relative risk in women than in men (6.17 vs. 3.36) (Table 3).

Table 1 The statistics of characters between the elderly group and younger group

\begin{tabular}{|c|c|c|c|c|}
\hline $\begin{array}{l}\text { Patient's group } \\
\text { Variables }\end{array}$ & All & Patients $\geq 65$ years & Patients $<65$ years & $P$ value \\
\hline Number & 290 & 146 & 144 & \\
\hline Age (in years) & $64.49 \pm 13.1$ & $75.4 \pm 6.6$ & $53.4 \pm 7.6$ & \\
\hline Female & $109(37.6 \%)$ & $71(48.6 \%)$ & $38(26.4 \%)$ & \\
\hline \multicolumn{5}{|l|}{ Cardiovascular Event History } \\
\hline Myocardial infarction & $40(13.8 \%)$ & $21(14.4 \%)$ & $19(13.2 \%)$ & 0.41 \\
\hline Angina pectoris & $87(30 \%)$ & $44(30.1 \%)$ & $43(29.9 \%)$ & 0.48 \\
\hline Tachycardia & $4(1.4 \%)$ & $2(1.4 \%)$ & $2(1.4 \%)$ & 0.49 \\
\hline Cardiovascular diseases* & $100(34.5 \%)$ & $57(39 \%)$ & $43(29.8 \%)$ & $<0.05$ \\
\hline Cardiac arrhythmia & $19(6.5 \%)$ & $12(8.2 \%)$ & $7(4.9 \%)$ & 0.18 \\
\hline Congestive heart failure* & $15(5.2 \%)$ & $13(8.9 \%)$ & $2(1.4 \%)$ & $<0.05$ \\
\hline Stroke or temporal ischemia* & $20(6.9 \%)$ & $16(10.9 \%)$ & $4(2.8 \%)$ & $<0.05$ \\
\hline Coronary artery bypass grafting surgery & $9(3.1 \%)$ & $6(4.1 \%)$ & $3(2.1 \%)$ & 0.27 \\
\hline Coronary angioplasty & $54(22.5 \%)$ & $30(20.5 \%)$ & $24(16.7 \%)$ & 0.35 \\
\hline \multicolumn{5}{|l|}{ Risk Factor } \\
\hline High blood pressure* & $176(60.7 \%)$ & $103(70.5 \%)$ & $73(50.7 \%)$ & $<0.05$ \\
\hline Diabetes* & $89(30.7 \%)$ & $53(36.3 \%)$ & $36(25 \%)$ & $<0.05$ \\
\hline Lipidemia* & $89(30.7 \%)$ & $36(24.6 \%)$ & $53(36.8 \%)$ & $<0.05$ \\
\hline Family history of Cardiovascular disease* & $68(23.4 \%)$ & $23(15.7 \%)$ & $45(31.2 \%)$ & $<0.05$ \\
\hline Smoker* & $64(22.1 \%)$ & $7(4.8 \%)$ & $57(39.6 \%)$ & $<0.05$ \\
\hline
\end{tabular}

Data: mean (standard deviation), number (percentage) *mean $P<0.05$ 
Table 2 Relation between MCB tests and Cardiovascular Events by Age

\begin{tabular}{|c|c|c|c|c|}
\hline \multicolumn{5}{|c|}{ Age (Patient Number) } \\
\hline \multirow{2}{*}{$\begin{array}{l}\text { Acute coronary } \\
\text { syndrome (ACS) }\end{array}$} & \multicolumn{2}{|c|}{$\geqq 65$ years $(N=146)$} & \multicolumn{2}{|c|}{$<65$ years $(N=144)$} \\
\hline & Positive & Negative & Positive & Negative \\
\hline MCB positive & 28 (19.2\%) & $62(42.4 \%)$ & 20 (13.9\%) & $16(11.1 \%)$ \\
\hline MCB negative & $7(4.8 \%)$ & 49 (33.5 \%) & $18(12.5 \%)$ & $9062.5 \%)$ \\
\hline Odds ratio & $3.161^{*}$ & & $6.25^{*}$ & \\
\hline
\end{tabular}

* mean $P<0.05$

The MCB tests in the two age groups demonstrated differences in sensitivity, specificity, and PPV $(p<0.05)$. The MCB tests also demonstrated improved sensitivity (80 \%) and NPV (87.5\%) for elderly patients, and higher specificity (84.9 \%) and PPV (55.6\%) for younger patients. Cardiac biomarkers exhibited a higher positive likelihood ratio $(\mathrm{LR}+)$ in the diagnosis of acute cardiovascular syndrome in young patients (3.49 vs. 1.43) (Table 4).

Differences were also observed in the sensitivity and PPV of MCB tests across genders. The biomarkers had higher sensitivity (80\%) and NPV (93.1\%) in female patients, while the specificity (66.1\%) and PPV (43.4\%) were higher in male patients. The $\mathrm{LR}+$ diagnosis of acute coronary syndrome was higher in female patients than in male patients (2.03 vs. 1.81 ) (Table 5$)$.

When the efficacy of MCB tests across both age and gender groups was investigated, $\mathrm{MCB}$ tests showed the highest sensitivity $(87.5 \%)$ and negative likelihood ratio (LR-) in elderly female patients, and the highest specificity (91.2\%), NPV (93.9\%), and LR+ (5.67) in young female patients (Table 6).

\section{Discussion}

Acute coronary syndrome is diagnosed on the basis of typical angina, electrocardiogram (ECG) results, and cardiac enzymes analysis. Patients with positive for two out of the three clinical criteria are diagnosed with acute coronary syndrome. This study focused on patients who

Table 3 Relation between MCB tests and Cardiovascular Events By Gender

\begin{tabular}{|c|c|c|c|c|}
\hline \multicolumn{5}{|c|}{ Gender (Patient Number) } \\
\hline \multirow{2}{*}{$\begin{array}{l}\text { Acute coronary } \\
\text { syndrome (ACS) }\end{array}$} & \multicolumn{2}{|c|}{ Male $(N=181)$} & \multicolumn{2}{|c|}{ Female $(N=109)$} \\
\hline & Positive & Negative & Positive & Negative \\
\hline $\begin{array}{l}\text { Cardiac biomarker } \\
\text { positive }\end{array}$ & $32(17.6 \%)$ & $44(24.3 \%)$ & $16(14.6 \%)$ & $35(32.1 \%)$ \\
\hline $\begin{array}{l}\text { Cardiac biomarker } \\
\text { negative }\end{array}$ & $21(11.6 \%)$ & $84(46.4 \%)$ & $4(3.6 \%)$ & $54(49.5 \%)$ \\
\hline Odds ratio & $3.364^{*}$ & & $6.171^{*}$ & \\
\hline
\end{tabular}

Table 4 Diagnostic Capabilities of MCB tests by Age

\begin{tabular}{llll}
\hline & \multicolumn{2}{l}{ Patient of Age } & \multirow{2}{*}{$P$ value } \\
\cline { 2 - 3 } & Patients $\geq 65$ years & Patients <65 years & \\
\hline Sensitivity & $80.0 \%(64.1-90.0)$ & $52.6 \%(37.3-67.5)$ & $<0.05^{*}$ \\
Specificity & $44.1 \%(35.3-53.4)$ & $84.9 \%(76.9-90.5)$ & $<0.05^{*}$ \\
PPV & $31.1 \%(22.5-41.3)$ & $55.6 \%(39.6-70.5)$ & $<0.05^{*}$ \\
NPV & $87.5 \%(76.4-93.8)$ & $83.3 \%(75.2-89.2)$ & 0.28 \\
LR+ & $1.43(1.13-1.81)$ & $3.49(2.02-6.00)$ & \\
LR- & $0.45(0.22-0.91)$ & $0.56(0.39-0.79)$ & \\
\hline
\end{tabular}

PS: PPV, NPV, LR+ and LR- mean positive and negative predictive values, positive and negative likelihood ratios, respectively

*mean $P<0.05$

experienced chest pain and who were suspected of having acute coronary syndrome. If the patient demonstrated ST-segment elevation and LBBB they were diagnosed with acute coronary syndrome. In such cases, cardiac enzyme analysis was not necessary. Patients who experience chest pain without any obvious change in their electrocardiogram or patients with atypical symptoms such as chest tightness, fainting spells, sweating, and shortness of breath are confounding for clinicians. In such cases, it is necessary to examine cardiac enzymes to aid in arriving at the proper diagnosis. Thus, we investigated the diagnostic efficacy of MCB tests in patients suspected of having acute coronary syndrome.

Rapid and accurate recognition together with efficient or rapid service strategy (e.g. same-day transfer) had been shown to increase the access rate to subsequent cath-lab management and further reduced hospital stay length in ACS patients. Based on this, an efficient and cost-effective diagnostic strategy prior to optimal implementation of health care model had become a major challenge in current clinical practice. For example, Campo et al. proposed a Hub and Spoke model in intermediate-to-high risk non-ST-segment elevation ACS patients referral, while other center ever reported [9]. Previous studies have demonstrated the effects of age and gender on the diagnosis of acute coronary syndrome. The use of electrocardiograms in

Table 5 Diagnostic Capabilities of MCB tests by Gender

\begin{tabular}{llll}
\hline & \multicolumn{2}{l}{ Patient of gender } & $P$ value \\
\cline { 2 - 3 } & Men $(N=181)$ & Women $(N=109)$ & \\
\hline Sensitivity & $61.1 \%(47.8-73.0)$ & $80.0 \%(58.4-91.9)$ & $<0.05^{*}$ \\
Specificity & $66.1 \%(57.5-73.8)$ & $60.7 \%(50.3-70.2)$ & 0.23 \\
PPV & $43.4 \%(32.9-54.6)$ & $31.4 \%(20.3-45.0)$ & 0.07 \\
NPV & $80 \%(71.4-86.5)$ & $93.1 \%(83.6-97.3)$ & $<0.05^{*}$ \\
LR+ & $1.81(1.31-2.49)$ & $2.03(1.45-2.85)$ & \\
LR- & $0.59(0.41-0.84)$ & $0.33(0.14-0.81)$ & \\
\hline
\end{tabular}

PS: PPV, NPV, LR+ and LR- mean positive and negative predictive values, positive and negative likelihood ratios, respectively *mean $P<0.05$ 
Table 6 Diagnostic Capabilities of MCB tests by Gender and Age

\begin{tabular}{|c|c|c|c|c|}
\hline & \multicolumn{2}{|c|}{$\geq 65$ years old $(N=146)$} & \multicolumn{2}{|c|}{$<65$ years old $(N=144)$} \\
\hline & Male $(N=75)$ & Female $(N=71)$ & Male $(N=106)$ & Female $(N=38)$ \\
\hline Sensitivity & 73.7 \% (51.2-88.2) & $87.5 \%(64-96.5)$ & $52.9 \%(36.7-68.5)$ & $50.0 \%(15.0-85.0)$ \\
\hline Specificity & $46.4 \%(34.0-59.3)$ & $41.8 \%(29.7-55.0)$ & $81.9 \%(71.5-89.1)$ & $91.2 \%(77.0-97.0)$ \\
\hline PPV & $31.8 \%(20.0-46.6)$ & $30.4 \%(19.1-44.8)$ & 58.1 \% (40.8-73.6) & $40.0 \%(11.8-76.9)$ \\
\hline NPV & 83.9 \% (71.1-89.2) & $92 \%(75.0-97.8)$ & 78.7 \% (68.1-86.4) & $93.9 \%(80.4-98.3)$ \\
\hline LR+ & $1.37(0.96-1.98)$ & $1.50(1.12-2.01)$ & $2.93(1.63-5.26)$ & $5.67(1.32-24.37)$ \\
\hline LR- & $0.57(0.25-1.26)$ & $0.29(0.08-1.13)$ & $0.57(0.39-0.83)$ & $0.55(0.20-1.47)$ \\
\hline
\end{tabular}

PS: PPV, NPV, LR+ and LR- mean positive and negative predictive values, positive and negative likelihood ratios, respectively

elderly patients can be problematic, such as in cases when the patient has a pacemaker or relatively low ST-segment elevation [10]. Elderly patients can exhibit symptoms similar to myocardial infarction, such as with gastritis or pneumonia [11-13]. Further, only a small proportion of elderly patients with acute coronary syndrome experience typical angina symptoms [14]. Finally, patients with congestive heart failure, diabetes, or coronary artery bypass graft surgery may exhibit atypical chest pains [14]. The current study also demonstrated that elderly patients are likely to exhibit these atypical factors. In addition, patients who smoke or have dyslipidemia exhibit relatively typical chest pain symptom, leading to many cases of acute coronary syndrome in younger patients [15].

Conventionally, female patients are less likely to seek for medical help than male patients. In addition, the symptoms of myocardial ischemia with chest pain are apparent atypically and easily confused with female chest wall or breast lesions with pain [15]. A relatively low proportion of electrocardiograms in women show STsegment elevation; thus, the use of biomarkers to analyze the probability of acute coronary syndrome in women can help overcome these difficulties in a more efficient manner. This study also demonstrated that young and female patients have a relatively higher risk of acute coronary syndrome after positive biodetection.

Troponin I is an important biomarker for diagnosing acute myocardial infarction due to its sensitivity and specificity in the detection of cardiomyocyte injury, as well as its character of remaining detectable in the blood for a continuous period of 4 days, and up to 2 weeks. However, there is also a shortcoming for clinical Troponin I use, for example, it only begins to rise $6 \mathrm{~h}$ after disease onset. However, patients with acute coronary syndrome will often seek for medical attention shortly after symptoms emerge, resulting in a time delay between the clinical symptoms and diagnostic results. McCord's et al. has reported that $49 \%$ of patients with acute coronary syndrome arrive at the hospital within $4 \mathrm{~h}$ of disease onset, leading to a large number of patients remain undiagnosed in the emergency room for hours due to the time gap as mentioned above [16].
According to a joint European Society of Cardiology (ESC) and American College of Cardiology (ACC) committee recommendation for diagnostic indicators of myocardial infarction, the 99th percentile upper range of troponin I in normal population serves as the recommended critical value for a diagnosis of myocardial infarction and myocardial damage; however, this may cause complications for an accurate diagnosis [17]. Although troponin I demonstrates high specificity, several factors, including renal failure, sepsis, chest trauma, and anthracycline use, may generate false-positive results due to elevated troponin I levels, resulting in erroneous treatment and unnecessary hospital stays [18].

In the current study MCB tests had higher specificity in elderly women than in men (Table 6). In particular, troponin I showed high specificity in female patients, which may help to clarify the real disease in a great portion of women manifested as angina clinically. In this regard, if a patient presented with abnormal troponin $I$ value, the possible misclassification as normal or false negative rate in women can be largely avoided and can be discharged from ED safely. Studies have shown that if only cardiac biomarkers are monitored within the first $6 \mathrm{~h}$ of onset of myocardial infarction, they are unable to provide sufficient diagnostic capability in terms of sensitivity and specificity [17]. Due to these aforementioned reasons, patients are unable to receive accurate diagnoses in a timely fashion, which not only results in overcrowded emergency rooms but also causes unnecessary hospital stays and significantly higher costs [18]. Of the 290 patients recruited in this study, $18.3 \%$ of the women experienced cardiovascular events, which is considerably lower than the $29.3 \%$ of men who experienced a cardiovascular event. Previous studies have demonstrated that the proportion of women with abnormal troponin I levels was relatively low because only approximately $6 \%$ of troponin $\mathrm{T}$ and $3 \%$ of troponin I exist in a free-floating state within the myocardial tissue of female gender. Since men typically have a greater number of cardiomyocytes than women, therefore the troponin I levels in men are easier to monitor or identified [19]. 
From the perspective of emergency room doctors, a quick and accurate diagnosis of acute coronary syndrome in elderly patients with chest pain is a major challenge. While patients' medical records and physiology can be conveniently examined, both these methods have lower sensitivity and specificity. Although electrocardiograms are continuously monitored and in general may have a diagnostic specificity of $92 \%$, their sensitivity is only $39 \%$ [20]. Several studies have shown that the use of $\mathrm{MCB}$ tests-such as troponin I, CK-MB, and myoglobin, which are related to cardiomyocyte necrosis, or BNP, which is related to cardiomyocyte function-can effectively improve the risk classification of patients with acute coronary syndrome. Due to the traditional shortcomings of individual biomarkers, the American College of Cardiology recommends that myoglobin be included in our MCB approach to improve diagnostic capability [21]. Since myoglobin is commonly present in skeletal muscle and myocardium, its levels will elevate 1-2 $\mathrm{h}$ after ACS symptoms. While myoglobin has a relatively low specificity, it has a very high sensitivity. Thus, if myoglobin is added as a biomarker for the identification of acute coronary syndrome, the diagnostic workflow may be executed more quickly and accurately. Of note, the proportion of female gender varied largely in different age groups (categorized as $<$, or $>=$ 65 years old) in our current work, which may potentiate the bias in the results generated.

By using a MCB approach we were able to effectively identify patients with low-risk chest pain. In particular, the MCB approach demonstrated excellent diagnostic capability in female and elderly patients, both of whom are considered to be challenging in accurate diagnosis. In our current work, both populations presented with relatively good sensitivity and NPV (>=80\%). In particular, the sensitivity and specificity for elderly female patients were near $90 \%$. In light of these findings, we can not only reduce the amount of time patients spend in the emergency room but also lessen the burden on attending clinicians.

One study showed that when 1285 emergency room chest pain patients were monitored for myoglobin, CK$\mathrm{MB}$, and troponin I over $90 \mathrm{~min}$, they achieved a NPV of $100 \%$. Of the 508 patients who were diagnosed as negative, only one returned to the emergency room with myocardial infarction 30 days after the diagnosis [22]. This demonstrates that multiple biomarker tests can effectively exclude patients with chest pains and potential acute coronary syndrome. Furthermore, non-myocardial infarction patients can be excluded when the elevation of the CK-MB level over $2 \mathrm{~h}$ exceeds $1.5 \mathrm{ng} / \mathrm{mL}$ or the troponin I level exceeds $0.2 \mathrm{ng} / \mathrm{mL}$ [23].

The current study demonstrates that a MCB approach is more effective in the evaluation of elderly patients than young patients. During the process of assessing and selecting potential groups and excluding potential cases, the elderly patient group showed high NPV (87.5\%) and sensitivity $(80 \%)$, both of which were statistically significant. These results showed that by using the MCB approach for elderly patients with chest pain, one can quickly and accurately exclude acute coronary syndrome and other cardiovascular diseases in $2 \mathrm{~h}$. Thus, the amount of time patients spend in the emergency room is reduced and unnecessary hospital stays are avoided. A recent study showed that a MCB approach can reduce the amount of time chest pain patients spend in the emergency room from 1 to 2 days to just 3-4 h [24]. Another study shows that by expediting the diagnostic process, the MCB approach can also lessen the costs of treatment for patients with chest pain [25]. Thus, this tool may aid in reducing emergency room crowding and minimize subsequent medical expenses [25].

Moreover, by using point-of-care monitoring MCB, we can speed up report completion time and therefore improve the efficacy of clinical practice. Studies have shown that as compared with traditional testing, the reporting time in point-of-care monitoring systems can be decreased by 75-85\% [26]. Such point-of-care monitoring systems are able to aid in expediting diagnosis.

\section{Conclusions}

Our results revealed that due to the high sensitivity and NPV of MCB, the clinical implementation of such diagnostic tools can help physicians exclude acute coronary syndrome quickly and accurately in elderly patients present with atypical chest pain in the emergency department. Furthermore, we also demonstrated that $\mathrm{MCB}$ tests are effective in the diagnosis of acute coronary syndrome in elderly and female patients. The clinical use of MCB in high-risk, atypical chest pain or in elderly patients can therefore facilitate accurate and proper diagnosis in a more timely fashion, reduce the time spent in the emergency room, and expedite treatment delievery. Finally, MCB tests may enormously improve the clinical workflow of emergency treatment and effectively reduces the rates of diagnostic misclassification encountered in certain challenging patient populations from the emergency room.

\section{Abbreviations \\ ACS: Acute coronary syndrome; BNP: Brain natriuretic peptide; CK-MB: Creatine kinase-myocardial band isoenzyme fraction; ECG: Electrocardiogram; \\ ED: Emergency department; LBBB: Left bundle branch block; MCB: Multiple cardiac biomarker; NSTEMI: Non-ST-segment elevation myocardial infarction; POCT: Point-of-care testing; STEMI: ST-segment elevation myocardial infarction}

\section{Acknowledgement}

None.

Funding

None. 


\section{Availability of data and materials}

Owing to local institutional regulation (which in this study was approved years ago and at that stage the authors did not apply for data spread or distribution out of the institution), together with the newly applied "Personal Information Protection Act" in Taiwan, the data will not be appropriate to be released in public place. The spread and data release will cause some concern from local ethical committee based on current institution regulations. Data are available from the "Mackay Memorial Hospital" Institutional Data Access/Ethics Committee for researchers who meet the criteria for access to confidential data.

The contact information as fllows:

Mackay Memorial Hospital

Address : No. 92, Sec. 2, Zhongshan N. Rd., Taipei City 10449, Taiwan TEL:02-25433535\#3486 3488, EMAIL:mmhirb82@gmail.com (Institutional Review Board)

\section{Authors' contributions}

WHC carried out the data collection and analysis, participated in the design alignment and drafted the manuscript. CLH carried out the edition and the draft of the manuscript. DKC participated in the manuscript drafting and the design of the study, performed the statistical analysis. SCS helped to revise the manuscript. All authors read and approved the final manuscript.

\section{Competing interests}

The authors declare that they have no competing interests.

\section{Consent for publication}

N/A.

\section{Ethics approval and consent to participate}

This study was approved by local ethical institutional committee (Mackay Memorial Hospital) for retrospective data analysis without informed consent of study participants (IRB No: 08CT1109). Data security was guaranteed and all authors had no access to patient identifying information before and after data analysis. Study participants involved in this study were not under clinical service of current study physicians or researchers.

\section{Author details}

'Department of Internal Medicine, Division of Cardiology, MacKay Memorial Hospital, Taipei, Taiwan. ${ }^{2}$ Department of Medicine, Mackay Medical College, New Taipei city, Taiwan. ${ }^{3}$ Emergency Department, MacKay Memorial Hospital, Taipei, Taiwan. ${ }^{4}$ Department of Nursing, Mackay Junior College of Medicine, Nursing and Management, Taipei, Taiwan. ${ }^{5}$ Graduate Institute of Injury Prevention and Control, Taipei Medical University, Taipei, Taiwan. ${ }^{6}$ Institute of Mechatronic Engineering, National Taipei University of Technology, Taipei, Taiwan. ${ }^{7}$ Department of Internal Medicine, Division of Gastroenterology, Mackay Memorial Hospital, Taipei, Taiwan. ${ }^{8}$ School of Medicine, Taipei Medical University, Taipei, Taiwan.

\section{Received: 25 April 2016 Accepted: 30 September 2016}

\section{Published online: 07 October 2016}

\section{References}

1. Mehta RH, Rathore SS, Radford MJ, Wang Y, Krumholz HM. Acute myocardial infarction in the elderly: differences by age. J Am Coll Cardiol. 2001;38:736-41.

2. Wang SW, Ren GC, Shio SF, Yu SY, Zhen FY. Acute myocardial infarction in elderly Chinese. A clinical analysis of 631 cases and comparison with 389 younger cases. Jpn Heart J. 1998;29:301-7.

3. Hochman JS, Tamis JE, Thompson TD, Weaver WD, White HD, Van de Werf F, et al. Sex, clinical presentation, and outcome in patients with acute coronary syndromes. Global Use of Strategies to Open Occluded Coronary Arteries in Acute Coronary Syndromes IIb Investigators. N Engl J Med. 1999;341:226-32.

4. Hochman JS, McCabe CH, Stone PH, Becker RC, Cannon CP, DeFeo-Fraulini $T$, et al. Outcome and profile of women and men presenting with acute coronary syndromes: a report from TIMI IIIB. TIMI Investigators. Thrombolysis in Myocardial Infarction. J Am Coll Cardiol. 1997;30:141-8.

5. Hasdai D, Porter A, Rosengren A, Behar S, Boyko V, Battler A. Effect of gender on outcomes of acute coronary syndromes. J Am Coll Cardiol. 2003;91:1466-8.

6. Larsen Al, Dickstein K. BNP in acute coronary syndromes: the heart expresses its suffering. Eur Heart J. 2004;25:1284-6.
7. Aviles RJ, Askari AT, Lindahl B, Wallentin L, Jia G, Ohman EM, et al. Troponin $T$ levels in patients with acute coronary syndromes, with or without renal dysfunction. N Engl J Med. 2002;346:2047-52.

8. Horwich TB, Hamilton MA, Fonarow GC. B-type natriuretic peptide levels in obese patients with advanced heart failure. J Am Coll Cardiol. 2006;47:85-90.

9. Campo G, Menozzi M, Guastaroba P, Vignali L, Belotti LM, Casella G, et al. Same-day transfer for the invasive strategy of patients with non-ST-segment elevation acute coronary syndrome admitted to spoke hospitals: Data from the Emilia-Romagna Regional Network. Eur Heart J Acute Cardiovasc Care. 2015. doi:10.1177/2048872615610867

10. Alexander KP, Roe MT, Chen AY, Lytle BL, Pollack Jr CV, Foody JM, et al. Evolution in cardiovascular care for elderly patients with non-ST-segment elevation acute coronary syndromes: results from the CRUSADE National Quality Improvement Initiative. J Am Coll Cardiol. 2005;46:1479-87.

11. Faisal MA, Russell RM, Samloff IM, Holt PR. Helicobacter pylori infection and atrophic gastritis in the elderly. Gastroenterology. 1990;99:1543-4.

12. Walston J, McBurnie MA, Newman A, Tracy RP, Kop WJ, Hirsch CH, et al. Frailty and activation of the inflammation and coagulation systems with and without clinical comorbidities: results from the Cardiovascular Health Study. Arch Intern Med. 2002;162:2333-41.

13. Majumdar SR, Kim N, Colman I, Chahal AM, Raymond G, Jen H, et al. Incidental vertebral fractures discovered with chest radiography in the emergency department: prevalence, recognition, and osteoporosis management in a cohort of elderly patients. Arch Intern Med. 2005;165:905-9.

14. Alexander KP, Newby LK, Cannon CP, Armstrong PW, Gibler WB, Rich MW, et al. Acute coronary care in the elderly, part I: Non-ST-segment-elevation acute coronary syndromes: a scientific statement for healthcare professionals from the American Heart Association Council on Clinical Cardiology: in collaboration with the Society of Geriatric Cardiology. Circulation. 2007;115:2549-69.

15. Hung C-L, Hou CJ-Y, Yeh H-I, Chang W-H. Atypical Chest Pain in the Elderly: The Prevalence, Possible Mechanisms and Prognosis. Int J Gerontol. 2010;4:1-6.

16. McCord J, Nowak RM, McCullough PA, Foreback C, Borzak S, Tokarski G, et al. Ninety-minute exclusion of acute myocardial infarction by use of quantitative point-of-care testing of myoglobin and troponin I. Circulation. 2001;104:1483-8.

17. Thygesen K, Alpert JS, White HD, Joint ESC/ACCF/AHAWHF Task Force for the Redefinition of Myocardial Infarction, Jaffe AS, Apple FS, et al. Universal definition of myocardial infarction. Circulation. 2007;116:2634-53.

18. Kelley WE, Januzzi $J$, Christenson RH. Increases of Cardiac Troponin in Conditions other than Acute Coronary Syndrome and Heart Failure. Clin Chem. 2009;55:2098-112.

19. Elsaesser A, Hamm CE. Acute coronary syndrome: the risk of being female. Circulation. 2004;109:565-7.

20. Bhuiya FA, Pitts SR, et al. Emergency Department Visits for Chest Pain and Abdominal Pain: United States, 1999-2008. NCHS Data Brief. 2010;43:1-8.

21. Anderson JL, Adams CD, Antman EM, Bridges CR, Califf RM, Casey Jr DE. ACC/AHA 2007 guidelines for the management of patients with unstable angina/non-ST-elevation myocardial infarction: a report of the American College of Cardiology/American Heart Association Task Force on Practice Guidelines (Writing Committee to Revise the 2002 Guidelines for the Management of Patients with Unstable Angina/Non-ST-Elevation Myocardial Infarction): developed in collaboration with the American College of Emergency Physicians, American College of Physicians, Society for Academic Emergency Medicine, Society for Cardiovascular Angiography and Interventions, and Society of Thoracic Surgeons. J Am Coll Cardiol. 2007:50:e1-e157.

22. Ng SM, Krishnaswamy P, Morissey R, Clopton P, Fitzgerald R, Maisel AS. Ninety-minute accelerated critical pathway for chest pain evaluation. Am J Cardiol. 2001;88:611-7.

23. Fesmire FM. Delta CK-MB Outperforms Delta Troponin I at 2 Hours During the ED Rule Out of Acute Myocardial Infarction. Am J Emerg Med. 2000;18:1-8.

24. Than M, Cullen L, Reid CM, Lim SH, Aldous S, Ardagh MW, et al. A 2-h diagnostic protocol to assess patients with chest pain symptoms in the Asia-Pacific region (ASPECT): a prospective observational validation study. Lancet. 2011;377:1077-84.

25. Birkhahn RH, Haines E, Wen W, Reddy L, Briggs WM, Datillo PA. Estimating the clinical impact of bringing a multimarker cardiac panel to the bedside in the ED. Am J Emerg Med. 2011;29:304-8.

26. Wu AHB. Point-of-Care Testing for Conventional Cardiac markers. Point Care. 2006;5:20-4 